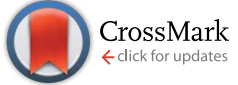

Cite this: RSC Adv., 2016, 6, 98748

Received 16th August 2016

Accepted 12th October 2016

DOI: $10.1039 / \mathrm{c} 6 \mathrm{ra20610g}$

www.rsc.org/advances

\section{A closer look at two-photon absorption, absorption saturation and nonlinear refraction in gold nanoclusters}

\author{
Joanna Olesiak-Banska, ${ }^{*}$ Magdalena Waszkielewicz, Katarzyna Matczyszyn \\ and Marek Samoc
}

\begin{abstract}
The optical properties of atomically-precise gold nanoclusters differ significantly from those of plasmonic gold nanoparticles, in both linear and nonlinear regimes. Here, we present a systematic study on the thirdorder nonlinear optical (NLO) properties of thiol-protected gold clusters in a wide range of wavelengths. We applied the $Z$-scan technique to evaluate the wavelength dispersion of two-photon absorption (2PA) and nonlinear refraction of the nanoclusters in water. The results are discussed with reference to 2PA of plasmonic gold nanoparticles and literature data on NLO properties of gold nanoclusters.
\end{abstract}

\section{Introduction}

Gold nanoclusters (NCs) are a new class of metallic nanomaterial, which consist of a certain number of central metal atoms surrounded by different types of ligands. Because of their sizes (1-2 nm), nanoclusters present a quantum-size effect, which leads to a discrete electronic structure. ${ }^{1}$ The spectra of NCs exhibit molecular-like one-electron transitions rather than collective excitation like in plasmonic gold nanocrystals. The unique electronic and geometric structure of nanoclusters gives rise to interesting combinations of properties like chirality, magnetism, redox chemistry etc. ${ }^{2-4}$ Thus, nanoclusters have gained great interest and applications in catalysis, photonics, biosensing and molecular electronics., ${ }^{\mathbf{5} 6}$

The gold nanoclusters were shown to exhibit significant optical nonlinearities. Because of their molecule-like electronic level structures one can expect that their NLO behavior, in particular, their nonlinear refraction and absorption (including two-photon absorption, 2PA) properties should be understood in the same terms as the analogous properties of molecular materials (see e.g. ref. 7 and 8). Several reports on third-order NLO properties of gold nanoclusters have been published and we summarize their results in Table 1. Multiphoton absorption and emission of nanoclusters have been usually investigated at single wavelengths, which presents only a fragmentary image of the NLO properties. The reported two-photon absorption crosssection $\sigma_{2}$ vary from several hundred GM to several hundred thousand GM. The discrepancies can be explained to some extent by various techniques and various measurement parameters applied. Especially, excitation with fs $v s$. ns laser

Advanced Materials Engineering and Modelling Group, Faculty of Chemistry, Wroclaw University of Science and Technology,Wroclaw, Poland.E-mail: joanna.olesiak@pwr. edu.pl pulses gives fundamentally different information about the NLO properties, as in the latter case cumulative effects may contribute significantly to the observed signal. Two-photon absorption (2PA) ${ }^{9}$ as well as saturable absorption $(\mathrm{SA})^{10}$ were observed for gold nanoclusters under ns excitation in the onephoton absorption (1PA) range of wavelengths. On the other hand, measurements with fs pulses are usually based on twophoton excited fluorescence (2PEF), which provide the twophoton action cross section values $\Phi \sigma_{2}$ (where $\Phi$ is the fluorescence quantum yield). ${ }^{11-13}$ The $\sigma_{2}$ values calculated from those measurements vary significantly from those measured directly, e.g. by using the $Z$-scan technique.

We present here a systematic investigation of third-order NLO properties of gold nanoclusters by the $Z$-scan technique with fs laser excitation in a broad range of wavelengths. We chose to investigate thiol-protected $\mathrm{Au}_{25}$ nanoclusters as those have been most widely described and applied. We identify the NLO processes observed in the sample as 2PA and SA, depending on the incident laser wavelength. We present for the first time the dispersion of both nonlinear refraction and twophoton absorption of gold nanoclusters. Thus, we provide a full description of third order NLO properties. Finally, we can compare the results to those reported in the literature for gold nanoclusters as well as other gold nanoparticles.

\section{Experimental}

\section{Nanoclusters synthesis}

Gold-captopril nanoclusters $\left(\mathrm{Au}_{25} \mathrm{Capt}_{18}\right)$ were prepared using a published procedure. ${ }^{14}$ Briefly, TOABr $(0.23 \mathrm{mmol}, 126.8 \mathrm{mg})$ was dissolved in $10 \mathrm{~mL}$ of methanolic solution of $\mathrm{HAuCl}_{4} \cdot 3 \mathrm{H}_{2} \mathrm{O}$ $(0.02 \mathrm{M})$ and after $20 \mathrm{~min}, 1 \mathrm{mmol}$ of captopril (217.2 $\mathrm{mg}$ ) was dissolved in $5 \mathrm{~mL}$ of methanol and rapidly added into the reaction mixture under stirring. After $30 \mathrm{~min}, 5 \mathrm{~mL}$ of aqueous 
Table 1 Summary of NLO properties of gold NCs

\begin{tabular}{|c|c|c|c|}
\hline Reference & Material $^{a}$ & Technique & Results \\
\hline Philip et al. ${ }^{9}$ & $\begin{array}{l}\mathrm{Au}_{25} \mathrm{SR}, \mathrm{Au}_{38} \mathrm{SR}, \mathrm{Au}_{144} \mathrm{SR}, \mathrm{Au} 4 \mathrm{~nm} \text {, } \\
\text { in toluene }\end{array}$ & $\begin{array}{l}\text { OA } Z \text {-scan, } 5 \mathrm{~ns}, 532 \mathrm{~nm}(T=25 \%) \text {, } \\
15 \mu \mathrm{J}\end{array}$ & $\begin{array}{l}\text { 2PA: } \mathrm{Au}_{25}: 2.0 \times 10^{-10} \mathrm{~m} \mathrm{~W}^{-1}, \mathrm{Au}_{38}: \\
3.5 \times 10^{-10} \mathrm{~m} \mathrm{~W}^{-1}, \mathrm{Au}_{144}: 7.5 \times \\
10^{-10} \mathrm{~m} \mathrm{~W}^{-1}\left(I=1.5 \times 10^{7} \mathrm{GW}\right. \\
\left.\mathrm{cm}^{-2}\right), \mathrm{Au} 5 \mathrm{~nm}: 1.5 \times 10^{-10} \mathrm{~m} \mathrm{~W}^{-1} \\
\left(I=2.2 \times 10^{7} \mathrm{GW} \mathrm{cm}^{2}\right)\end{array}$ \\
\hline Ding et al. ${ }^{10}$ & AuNC in water & OA $Z$-scan, $4.8 \mathrm{~ns}, 532 \mathrm{~nm}, 5 \mathrm{kHz}$ & $\begin{array}{l}\text { SA: } 0.029-0.07 \mathrm{GW} \mathrm{cm}^{2} \text { for } P=2-3.7 \\
\mathrm{~mW}\end{array}$ \\
\hline Oh et al. ${ }^{12}$ & AuNC-PEG $1.5 \mathrm{~nm} \pm 0.3 \mathrm{~nm}$ water & $\begin{array}{l}2 \mathrm{PEF}, 850-1300 \mathrm{~nm}, \approx 80 \mathrm{fs}, 250 \\
\mathrm{kHz}\end{array}$ & $\begin{array}{l}\text { 2PA: decreasing from } 670 \mathrm{GM} \text { at } 870 \\
\mathrm{~nm} \text {, to } 130 \mathrm{GM} \text { at } 1300 \mathrm{~nm}\end{array}$ \\
\hline Liu et al. ${ }^{11}$ & $\mathrm{Au}_{11} \mathrm{MUA} 1.7 \times 10^{-4} \mathrm{M}$, water & $\begin{array}{l}2 \mathrm{PEF} 800 \mathrm{~nm}, \mathrm{MHz} \text { and OA } Z \text {-scan } \\
800 \mathrm{~nm}, 1 \mathrm{kHz}, 180 \mathrm{fs}\end{array}$ & $\begin{array}{l}\text { 2PA: } 3426 \mathrm{GM}(800 \mathrm{~nm}, \mathrm{TPEF}) 8761 \\
\text { GM }(800 \mathrm{~nm}, \mathrm{OA} Z \text {-scan })\end{array}$ \\
\hline This work & $\mathrm{Au}_{25} \mathrm{Capt}_{18}$, water & $\begin{array}{l}\text { CA and OA } Z \text {-scan } 550-1100 \mathrm{~nm}, 130 \\
\text { fs, } 1 \mathrm{kHz}, 35 \text { to } 180 \mathrm{GW} \mathrm{cm}^{-2}\end{array}$ & $\begin{array}{l}\text { 2PA and SA: } \sigma_{2}=23800 \mathrm{GM}, 830 \\
\mathrm{GM} \text { and } 1510 \mathrm{GM} \text { at } 550 \mathrm{~nm}, 800 \mathrm{~nm} \\
\text { and } 900 \mathrm{~nm}\end{array}$ \\
\hline
\end{tabular}

${ }^{a}$ Abbreviations: SR $=\mathrm{SCH}_{2} \mathrm{CH}_{2} \mathrm{Ph}$, polyethylene glycol (PEG), mercaptoundecanoic acid (MUA), glutathione (SG), 1-dodecanethiol (DTT), 2naphthalenethiol (NT), 4-methylbenzenethiol (mBT), $\alpha$-toluenethiol $(\alpha-\mathrm{TT})$.

solution of $\mathrm{NaBH}_{4}(0.4 \mathrm{M})$, cooled to $4{ }^{\circ} \mathrm{C}$, was rapidly poured to the reaction mixture under vigorous stirring. The solution was allowed to react for $8 \mathrm{~h}$ in ice bath. The suspension was centrifuged. The clusters were repeatedly washed with methanol and ethanol. The final precipitate was dried in a water bath.

\section{$Z$-Scan measurements}

The $Z$-scan measurements were performed as described previously, with some modifications. ${ }^{15}$ A Quantronix Integra-C regenerative amplifier operating as an $800 \mathrm{~nm}$ pump and a Quantronix-Palitra-FS optical parametric amplifier were used to deliver wavelength tunable pulses with the duration $<130 \mathrm{fs}$, and a repetition rate of $1 \mathrm{kHz}$. The excitation beam with intensities ranging from 35 to $180 \mathrm{GW} \mathrm{cm} \mathrm{cm}^{-2}$ was used for simultaneous recording of open aperture (OA) and closedaperture (CA) Z-scan transmittance curves. Three Si photodiodes (Thor Laboratories Inc.) monitored the laser input, the OA signal, and the CA signal. An aqueous solution of nanoclusters $\left(c=11 \mathrm{mg} \mathrm{mL}^{-1}\right)$ was placed in $1 \mathrm{~mm}$ path length Starna sealed glass cuvettes. A reference cuvette containing a pure solvent was always measured under identical conditions to enable extraction of nonlinear refraction of the nanoclusters from the overall response of the sample. No nonlinear absorption of the solvent was observed in the range of wavelengths and intensities employed.

\section{Results and discussion}

The 1PA spectrum of the studied nanoclusters $\mathrm{Au}_{25} \mathrm{Capt}_{18}$ presents the clearly resolved absorption bands in the NIR and visible range, which indicate that the synthesis yielded a monodisperse solution of NCs. The bands positions at 800 $\mathrm{nm}, 675 \mathrm{~nm}, 550 \mathrm{~nm},(505 \mathrm{~nm}), 445 \mathrm{~nm},(400 \mathrm{~nm})$ correspond very well with theoretical and experimental spectra of $\mathrm{Au}_{25}(\mathrm{SR})_{18}$ found in the literature (see Fig. 2, gray solid line). ${ }^{16}$

We performed OA and CA Z-scan measurements on a water dispersion of $\mathrm{Au}_{25} \mathrm{Capt}_{18}$ in the range of wavelengths 550-1100 nm. Fig. 1 shows examples of OA and CA traces at several wavelengths. For all the wavelengths but $550 \mathrm{~nm}$ the CA traces present a decrease followed by an increase of transmittance, which is characteristic for a material with a positive (selffocusing) refractive nonlinearity (Fig. 1a). At $550 \mathrm{~nm}$ an opposite trend was observed, which implies overall negative refractive nonlinearity of the sample (including contributions from the cell walls, the solvent and the solute). OA traces showed a decrease of the transmittance in the range of wavelengths $>$ $775 \mathrm{~nm}$ and <650 nm (Fig. 1b). However, an increase of the transmittance and combination of the increase followed by the decrease in the focus were observed when $\lambda=650-750 \mathrm{~nm}$ were applied (Fig. 1c). It has to be noted that the nanoclusters were sensitive to the high intensity illumination, especially when the laser wavelength falls into the range of wavelengths where 1PA is observed. Excessively high intensities resulted in a photochemical transformation in clusters, identifiable as a deformation of $Z$-scan traces: a characteristic asymmetry of the OA traces (Fig. 1d).

We analyzed the collected data using the equations derived by Sheik-Bahae et al. ${ }^{17}$ The nonlinear refractive index $n_{2}$ and two-photon absorption coefficient $\alpha_{2}$ of the solutions were obtained from the nonlinear phase shift and the changes of transmittance of light passing through the sample. We 

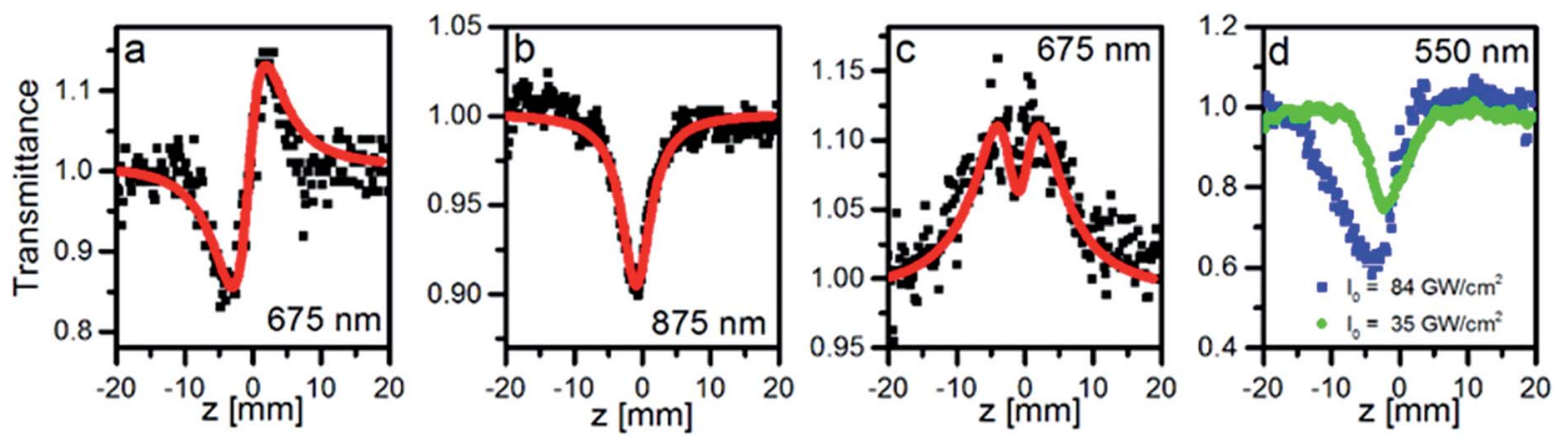

Fig. 1 Representative closed-aperture (CA) and open-aperture (OA) Z-scan traces of AuCapt (black dots - experimental data, red lines theoretical fits). Fitting of $C A$ and $O A$ assuming two-photon absorption was performed for (a) and (b). (c) Fitting of OA traces performed assuming a saturable absorption or a combination of 2PA and saturation of $1 \mathrm{PA}$. (d) Deformation of OA trace at $550 \mathrm{~nm}$ for excessively increased $I_{0}$ (intensity at the focus of the $Z$-scan setup).

calculated the NLO parameters of the solute assuming that the nonlinear contributions of the solvent and the solute sum up and the Lorentz local field approximation is valid. ${ }^{18}$

Two alternate ways of presenting the microscopic, concentration-independent cubic nonlinearities exist, either by quoting the real and imaginary parts of the second hyperpolarizability (third-order polarizability) of a species, $\operatorname{Re}[\gamma]$ and $\operatorname{Im}[\gamma]$, respectively, or by providing appropriate cross sections which are a measure of the nonlinear refraction and nonlinear absorption contributions. As proposed in ref. 19 we present here the spectra of $\sigma_{2}$ of a single nanocluster and the crosssection characterizing the nonlinear refraction $\sigma_{\mathrm{R}}$. Presentation of a single nanoparticle-oriented values of both refractive and absorptive part of the nonlinearity gives the basis for understanding NLO effects in gold nanoclusters. Also, it allows for quantitative comparison of NLO parameters between different types of nanoparticles and other materials. Fitting of the $Z$-scan traces led to $\sigma_{\mathrm{R}}$ and $\sigma_{2}$ spectra presented in Fig. 2 . The $\sigma_{\mathrm{R}}$ is negative in the whole range of wavelengths, with the magnitude increasing rapidly at shortest wavelengths up to $-40000 \mathrm{GM}$ at $550 \mathrm{~nm}$. 2PA bands at $\sim 800$ and $\sim 900 \mathrm{~nm}$ are clearly visible, with $\sigma_{2}=850$ and $1500 \mathrm{GM}$, respectively. These

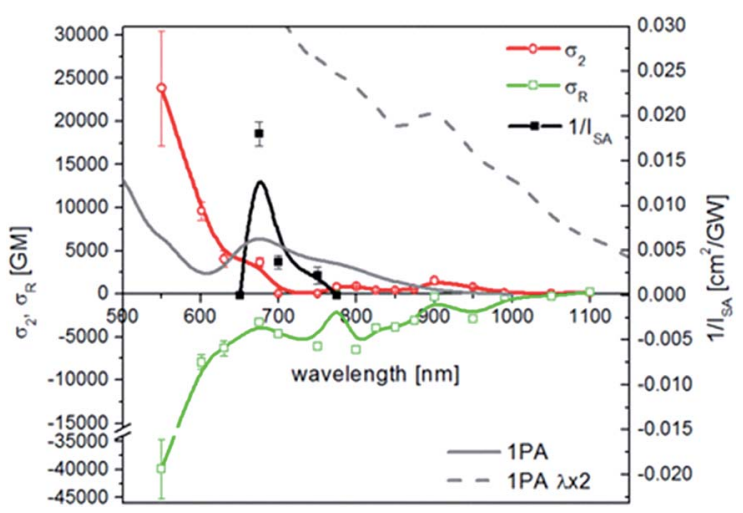

Fig. 2 2PA spectrum (red line) and nonlinear refraction spectrum (green line), 1PA (gray solid line), and 1PA at twice the wavelength (gray dashed line) together with the reciprocal of the saturation intensity (black line). bands can be related to the d-sp interband transitions located at 400-450 nm in the 1PA spectrum. ${ }^{20}$ Additionally, in 550-650 nm range a strong 2PA was observed with $\sigma_{2}$ increasing up to 24000 GM. Resonant excitation in the range of 1PA implies possible excited state absorption mechanism of this transition.

To reproduce the increase of the transmittance observed in OA $Z$-scans for $\lambda=650-750 \mathrm{~nm}$ we had to introduce to our calculations an absorption saturation effect. Several models of SA in metal nanoparticles has been reported, including 1PA saturation and 2PA. ${ }^{10,15,21}$ In case of gold nanoclusters, they present complex 1PA spectra with mixed contributions of core and surface states. ${ }^{20} \mathrm{~A}$ simplified three-level energy diagram can be applied, which consists of a ground state $\mathrm{S}_{0}$, the first and second excited states $S_{1}$ and $S_{2}$, respectively, (as $\mathrm{Au}_{25} \mathrm{Capt}_{18}$ nanoclusters exhibit long photoluminescence decay times, on the order of a few $\mu$ s (ref. 22) triplet states may be in fact involved). Relaxation from the higher-lying singlet and triplet states is rapid, thus the population densities of these states are very small and can be ignored.

The rate equation of the excited state $S_{1}$ and the equation describing changes of the absorption of the incident light are:

$$
\begin{gathered}
\frac{\partial N_{1}}{\partial t}=\frac{\sigma_{0} N_{0} I}{h \nu}-\frac{N_{1}}{\tau_{1}} \\
\frac{\mathrm{d} I}{\mathrm{~d} z}=-\sigma_{0} N_{0} I-\sigma_{1} N_{1} I
\end{gathered}
$$

where $\sigma_{0}$ is a 1PA cross section, $\tau_{1}$ is a total relaxation time of the $\mathrm{S}_{1}$ state (including intersystem crossing) and $N_{0}, N_{1}$ are population densities of levels $S_{0}, S_{1}$, respectively. In the case of ultrafast pulsed laser excitation (pulse width $\tau_{\mathrm{p}}$ in the fs range), the $\tau_{\mathrm{p}} \ll \tau_{1}$ and the steady state is determined by the pulse duration. The eqn (1) and (2) can be solved and give a formula for homogenously broadened saturable absorption $\alpha_{\mathrm{SA}}$ :

$$
\alpha_{\mathrm{SA}}=\frac{\alpha_{0}}{1+\frac{I}{I_{\mathrm{SA}}}}
$$

where $\alpha_{0}$ is the one-photon absorption coefficient and $I_{\mathrm{SA}}$ is the saturation intensity defined as $h \nu \sigma_{0}{ }^{-1} \tau_{\mathrm{p}}{ }^{-1}$. Finally, the 
transmittance $(T)$ changes with the distance of the light propagation are described as:

$$
\frac{\mathrm{d} I}{\mathrm{~d} z}=\alpha_{\mathrm{SA}} I+\alpha_{2} I^{2}
$$

The formula accounts for an interplay between SA and 2PA, which, when present simultaneously, result in the OA trace, where a decrease of $T$ at the focus of the beam is superimposed on the broader increase. Eqn (4) was applied for fitting OA traces at $\lambda=650-750 \mathrm{~nm}$ and good reproduction of experimental results was obtained. The calculated $1 / I_{\mathrm{SA}}$ values are presented in Fig. 2.

Table 1 shows the NLO coefficients for Au NCs reported in the literature and the present results. Experiments with fs pulsed lasers (usually 2PEF) gave $\sigma_{2}$ values ranging from $427000 \mathrm{GM}$ down to $670 \mathrm{GM}$ at $\sim 800 \mathrm{~nm}$. On the other hand, when the $Z$-scan technique with ns lasers was applied, contradicting results were reported for the same wavelength, either 2PA or SA.9,10 No $\sigma_{2}$ values were calculated, and $\alpha_{2}$ was determined at $532 \mathrm{~nm}$ to be from $2.0 \times 10^{-10} \mathrm{~m} \mathrm{~W}^{-1}$ for $\mathrm{Au}_{25}$ up to $7.5 \times 10^{-10} \mathrm{~m} \mathrm{~W}^{-1}$ for $\mathrm{Au}_{144}$. Liu et al. compared $\sigma_{2}$ measured with $Z$-scan and 2 PEF methods and obtained values of $8761 \mathrm{GM}$ and 3426 GM, respectively. ${ }^{\mathbf{1 1}}$ Note that the present report is the first to provide the full, wide-wavelength-range spectral characterization of the NLO properties of the NCs.

In line with our previous results, we can determine certain figures of merit for the NLO properties of NCs and compare them with those for other gold nanoparticles. A convenient figure of merit is $\sigma_{2}$ scaled by the object molar mass. For gold nanorods (aspect ratio 3.4, $\lambda_{\mathrm{l}-\mathrm{SPR}}=845 \mathrm{~nm}, \sigma_{2}$ at $550 \mathrm{~nm}$ ) and gold nanoshells (120 nm silica nanospheres with $\sim 10 \mathrm{~nm}$ gold shell, $\sigma_{2}$ at $600 \mathrm{~nm}$ ) we obtained values of 7.96 and 2.56, respectively (in $\left.\mathrm{GM} \mathrm{g} \mathrm{mol}{ }^{-1}\right) .^{15,21,25}$ In comparison, for $\mathrm{Au}_{25}(-$ Capt $)_{18}$ at $550 \mathrm{~nm}$ we measured $\sigma_{2} / M_{\mathrm{w}}=2.69$, thus the NCs are good two-photon absorbers, comparable to gold nanoshells, although they contain a significantly lower gold content. In comparison with the best two-photon absorbing organic molecules, nanoclusters exhibit significantly higher $\sigma_{2}$ and $\sigma_{2} / M_{\mathrm{w}}$ values (e.g. for an $\mathrm{N}$-cored Ru-containing dendrimer, $\sigma_{2}=$ $10600 \mathrm{GM}$ and $\sigma_{2} / M_{\mathrm{w}}=1.13$ at $\left.580 \mathrm{~nm}\right){ }^{26}$

\section{Conclusions}

In summary, we measured for the first time the full two-photon absorption spectrum and dispersion of nonlinear refraction of thiol-stabilized gold nanoclusters in water. Very strong 2PA was seen in the range of 1PA wavelengths, with $\sigma_{2}$ up to $24000 \mathrm{GM}$. Other 2PA bands of lower intensity at 800 and $900 \mathrm{~nm}$ were characterized by cross sections of 830 GM and 1510 GM, respectively. Nonlinear refraction cross-section values were negative in the whole range of wavelengths. Moreover, SA effects were found to dominate when the incident wavelength was from 650 to $750 \mathrm{~nm}$. Efficient 2PA makes gold nanoclusters good candidates for optical limiting applications, and, combined with their fluorescent properties, a marker for multiphoton imaging.

\section{Acknowledgements}

This work was supported by the National Science Centre under grant DEC-2013/10/A/ST4/00114, DEC-2013/09/B/ST5/03417 and by a statutory activity subsidy from the Polish Ministry of Science and Higher Education for the Faculty of Chemistry of Wroclaw University of Technology.

\section{References}

1 R. C. Jin, Nanoscale, 2010, 2, 343.

2 S. Knoppe and T. Burgi, Acc. Chem. Res., 2014, 47, 1318.

3 W. Luo, S. J. Pennycook and S. T. Pantelides, Nano Lett., 2007, 7, 3134 .

4 H. Tsunoyama, H. Sakurai, Y. Negishi and T. Tsukuda, J. Am. Chem. Soc., 2005, 127, 9374.

5 P. Schwerdtfeger, Angew. Chem., Int. Ed., 2003, 42, 1892.

6 L. Shang, S. Dong and G. U. Nienhaus, Nano Today, 2011, 6, 401.

7 M. G. Kuzyk, K. D. Singer and G. I. Stegeman, Adv. Opt. Photonics, 2013, 5, 4.

8 F. Terenziani, C. Katan, E. Badaeva, S. Tretiak and M. Blanchard-Desce, Adv. Mater., 2008, 20, 4641.

9 R. Philip, P. Chantharasupawong, H. F. Qian, R. C. Jin and J. Thomas, Nano Lett., 2012, 12, 4661.

10 S. J. Ding, F. Nan, D. J. Yang, X. L. Liu, Y. L. Wang, L. Zhou, Z. H. Hao and Q. Q. Wang, Sci. Rep., 2015, 5, 9735.

11 C. L. Liu, M. L. Ho, Y. C. Chen, C. C. Hsieh, Y. C. Lin, Y. H. Wang, M. J. Yang, H. S. Duan, B. S. Chen, J. F. Lee, J. K. Hsiao and P. T. Chou, J. Phys. Chem. C, 2009, 113, 21082. 12 E. Oh, F. K. Fatemi, M. Currie, J. B. Delehanty, T. Pons, A. Fragola, S. Lévêque-Fort, R. Goswami, K. Susumu, A. L. Huston and I. L. Medintz, Part. Part. Syst. Charact., 2013, 30, 453.

13 I. Russier-Antoine, F. Bertorelle, M. Vojkovic, D. Rayane, E. Salmon, C. Jonin, P. Dugourd, R. Antoine and P. F. Brevet, Nanoscale, 2014, 6, 13572.

14 S. Kumar and R. Jin, Nanoscale, 2012, 4, 4222.

15 J. Olesiak-Banska, M. Gordel, R. Kolkowski, K. Matczyszyn and M. Samoc, J. Phys. Chem. C, 2012, 116, 13731.

16 M. Zhu, C. M. Aikens, F. J. Hollander, G. C. Schatz and R. Jin, J. Am. Chem. Soc., 2008, 130, 5883.

17 E. W. V. Stryland and M. Sheik-Bahae, Z-Scan measurements of optical nonlinearities, in Characterization techniques and tabulations for organic nonlinear optical materials, ed. M. G. Kuzyk and C. W. Dirk, Marcel Dekker, New York, 1998, pp. 655-692.

18 M. Samoc, A. Samoc, B. Luther-Davies, M. G. Humphrey and M. S. Wong, Opt. Mater., 2003, 21, 485.

19 M. Balu, L. A. Padilha, D. J. Hagan, E. W. Van Stryland, S. Yao, K. Belfield, S. Zheng, S. Barlow and S. Marder, J. Opt. Soc. Am. B, 2008, 25, 159.

20 C. M. Aikens, J. Phys. Chem. Lett., 2011, 2, 99.

21 M. Gordel, J. Olesiak-Banska, R. Kolkowski, K. Matczyszyn, M. Buckle and M. Samoc, J. Mater. Chem. C, 2014, 2, 7239. 22 Z. K. Wu and R. C. Jin, Nano Lett., 2010, 10, 2568. 
23 G. Ramakrishna, O. Varnavski, J. Kim, D. Lee and T. Goodson, J. Am. Chem. Soc., 2008, 130, 5032.

24 Y. Hamanaka, N. Okada, K. Fukagawa, A. Nakamura, Y. Tai and J. Murakami, J. Phys. Chem. C, 2012, 116, 10760.
25 M. Gordel, R. Kolkowski, J. Olesiak-Banska, K. Matczyszyn, M. Buckle and M. Samoć, J. Nanophotonics, 2014, 9, 093797. 26 R. L. Roberts, T. Schwich, T. C. Corkery, M. P. Cifuentes, K. A. Green, J. D. Farmer, P. J. Low, T. B. Marder, M. Samoc and M. G. Humphrey, Adv. Mater., 2009, 21, 2318. 